\title{
Diacronie
}

Studi di Storia Contemporanea

$N^{\circ} 22,2$ | 2015

Costruire

\section{Le comunità ebraiche in Australia e gli arrivi dall'Europa orientale fra XIX e XX secolo}

\section{Giuseppe Motta}

\section{(2) OpenEdition}

12 Journals

\section{Edizione digitale}

URL: http://journals.openedition.org/diacronie/1987

DOI: 10.4000/diacronie.1987

ISSN: 2038-0925

\section{Editore}

Association culturelle Diacronie

\section{Notizia bibliografica digitale}

Giuseppe Motta, "Le comunità ebraiche in Australia e gli arrivi dall'Europa orientale fra XIX e XX secolo », Diacronie [Online], № 22, 2 | 2015, documento 2, online dal 01 juin 2015, consultato il 09 octobre 2020. URL : http://journals.openedition.org/diacronie/1987 ; DOI : https://doi.org/10.4000/ diacronie. 1987 


\section{Diacronie}

N. 22 | 2|2015 Costruire. Rappresentazioni, relazioni, comunità

\section{2/}

\section{Le comunità ebraiche in Australia e gli arrivi dall'Europa orientale fra XIX e XX secolo}

Giuseppe MOTTA*

L'articolo si propone di analizzare la politica migratoria australiana nei confronti degli ebrei in fuga dall'Europa orientale e dalle violenze iniziate con la prima guerra mondiale e proseguite con la guerra civile russa, la guerra russo-polacca e l'avvento di una diffusa, seppur meno violenta, politica di discriminazione legale nei confronti delle comunità ebraiche in paesi come Romania e Polonia. Attraverso una ricerca condotta presso gli archivi nazionali australiani si è quindi cercato di capire quali siano stati le diverse risposte a tale emergenza umanitaria e vedere come un paese geograficamente extra-europeo ma culturalmente e politicamente legato al Vecchio Continente abbia reagito all'incremento delle domande di accoglienza da parte di una particolare categoria di migranti, profughi e rifugiati.

\section{Introduzione. In fuga dalla Russia zarista}

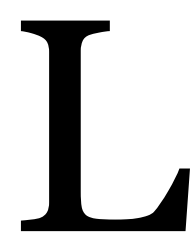

e condizioni di vita degli ebrei nella Russia zarista sono inevitabilmente condizionate dalla costituzione della Zona di Residenza, dove le comunità ebraiche vengono concentrate fin dall'epoca di Caterina la Grande e delle Spartizioni della Polonia nel XVIII secolo. La Zona di Residenza abbraccia il confine occidentale della Russia zarista: quasi l'intera Ucraina, la Bessarabia, la Polonia russa e le province lituane e bielorusse e ospita quasi per intero la popolazione ebraica in Russia. 2.600.000 abitanti in Ucraina e 1.400.000 nelle province di Lituania 
e Russia Biancaํ. La precaria condizione della realtà ebraica è aggravata inoltre da una serie di disposizioni di Nicola I come quelle relative al reclutamento forzato all'interno dell'esercito e delle scuole militari "canoniste", e dalle misure prese dopo la morte dello zar Alessandro II, nel 1881, che viene addebitata ai rivoluzionari ebraici e porta a un ulteriore peggioramento della situazione ${ }^{2}$. In tale contesto di repressione rientra la promulgazione delle Leggi di Maggio del 1882, che prevedono, fra l'altro, una serie di divieti di natura economica e che verranno seguite da ulteriori misure repressive come l'espulsione dalle città di Kiev (1886) e di Mosca (1891).

Gli ebrei, abbastanza naturalmente, reagiscono alle lunghe e pesanti discriminazioni mostrando chiare simpatie verso i nascenti movimenti socialisti rivoluzionari, come il Bund o il partito social-democratico di ispirazione marxista. Tali simpatie si rivelano controproducenti e rafforzano l'immagine dell'ebreo traditore che trama contro la Russia per sovvertire il potere costituito e impadronirsi del mondo. Questo messaggio viene propagandato con la falsificazione del libello dei Protocolli dei Savi di Sion, commissionato dalla polizia zarista e pubblicato nel 1903 su «Znamya», un giornale vicino al movimento ultra-conservatore e anti-semita dalle Centurie Nere3.

Negli stessi anni si verifica il tragico episodio del pogrom di Kishinev, dove in occasione della pasqua ebraica del 1903 si registra un barbarico eccidio che si conclude con 45 morti, centinaia di feriti e il saccheggio di migliaia di case e negozi da parte della folla inferocita. Le notizie del pogrom creano scompiglio nelle vicine località, per esempio a Varsavia dove pare che la popolazione ebraica organizzi delle squadre di autodifesa o a Derlav, Bakau, Galaţi, Tulcea e Buhuşi (Bessarabia) dove secondo il «Jewish Herald» russi e romeni sembrano iene assetate di sangue 4.

I pogrom si ripetono negli anni seguenti e hanno come effetto principale quello di costringere molti ebrei a lasciare la Russia per stabilirsi soprattutto negli Stati Uniti, dove si è costituito un solido network di organizzazioni di assistenza e solidarietà. L'emigrazione negli Stati Uniti nel periodo 1881-1910 è in costante crescita e una parte consistente di tale flusso proviene proprio dall'Europa orientale: nel 1900-1901 a New

\footnotetext{
${ }^{1}$ Memorandum del Joint Distribution Committee The Pale of Settlement in the Former Russian Empire, fondo USSR: Agro-Joint, Publicity, 1925-1927, 1921-1932, item 359421

${ }^{2}$ Questa politica venne poi conclusa durante la stagione delle Grandi Riforme di Alessandro II, nel 1856. DOMINICH, Larry, The Cantonists. The Jewish Children's Army of the Tsar, Jerusalem, Devora Publishing Company, 2003.

3 Il movimento delle Centurie Nere abbraccia gli ideali conservatori dello zarismo, opponendosi radicalmente ai diversi nazionalismi che fra il XIX e il XX si consolidano nei territori russi. LAQUER, Walter, Black Hundred: The Rise Of The Russian Extreme Right, New York, HarperCollins, 1993; DE MICHELIS. Cesare, Il manoscritto inesistente. I «Protocolli dei savi di Sion», Venezia, Marsilio, 1998.

4 «Jews in Romania and Poland Alarmed», in The New York Times, 21 maggio 1903.
} 
York, su un totale di 44.208 ebrei 24.927 vengono dalla Russia, 5.613 dalla Romania, 13.004 dall'Austria5.

Un altro importantissimo movimento che viene alimentato grazie a tali flussi è quello verso la Palestina, dove si costituiscono i primi piccoli insediamenti ebraici seguendo le linee della nascente dottrina sionista promossa da Moses Montefiore, Theodor Herzl e dal primo congresso dell'Organizzazione mondiale sionista, tenutosi a Basilea nel 1897.

Oltre ai pogrom, che si ripetono negli anni successivi al 1903, destano grandi preoccupazioni altri numerosi episodi, come il caso Beilis del 1911-13 - molto simile all'analogo affaire Dreyfus (Menahem Beilis è invece accusato di avere rapito e ucciso un ragazzo di tredici anni di Kiev) - e la "guerra" del 1912, che oppone polacchi ed ebrei in merito all'elezione di un deputato alla Duma e che scatena un duro boicotaggio a danno degli interessi economici ebraici ${ }^{6}$.

La precarietà delle condizioni dell'ebreo russo trova una perfetta descrizione nell'opera letteraria di Joseph Roth e nel suo Giobbe, la cui fede in Dio è messa alla prova dalle avversità e dalla disperazione della realtà della Russia zarista7. La vita all'interno della Zona di Residenza è invece descritta da Mary Antin nel libro The Promised Land del 1912, in cui si racconta come una bambina della Zona di Residenza veda il mondo diviso in due: il suo villaggio, Polotzk, e uno strano paese chiamato Russia:

I Knew that Polotzk and Vitebsk and Vilna and some other places were grouped together as the Pale of Settlement, and within this area the Czar commanded me to stay, with my father and mother and friends, and all other people like us. We must not be found outside the Pale, because we were $\mathrm{Jews}^{8}$.

5 JOSEPH, Samuel, Jewish Immigration to the United States from 1881 to 1910, New York, Columbia University, 1914, p. 172; WEINRYB, Bernard D., «East European Immigration to the United States», in The Jewish Quarterly Review, New Series, 45, 4/1955, p. 519; American Jewish Year Book, Vol. 3 (1901-1902), New York, JPS, 1902, p. 158.

${ }^{6}$ Sul caso Beilis e la suo impatto internazionale, cfr. BERKOWITZ, Joel, «The 'Mendel Beilis' Epidemic on the Yiddish Stage», in Jewish Social Studies, 8, 1/2001, p. 199. SAMUEL, Maurice, Blood Accusation: The Strange History of the Beilis Case, New York, A.A.Knopf, 1966.

7 Altrettanto doverosa è la citazione dell'opera di MAGRIS, Claudio, Lontano da dove. Joseph Roth e la tradizione ebraico-orientale, Torino, Einaudi, 1989.

${ }^{8}$ ANTIN, Mary, The Promised Land, Boston-New York, Cambridge, Riverside Press, 1912, pp. 1 et seq. 


\section{Le comunità ebraiche in Australia}

I flussi migratori in uscita dalla Russia sono sì principalmente diretti negli Stati Uniti ma percorrono anche strade meno battute verso paesi come Argentina, Canada o Sud Africa, ove si dirigono numerose comunità provenienti dall'Europa. Fra queste destinazioni c'è anche l'Australia, immenso continente che ha conosciuto un processo di colonizzazione solo dopo l'arrivo del capitano inglese James Cook, nel 1770, e che è stato poi inglobato nel Regno Unito, rimanendo però in gran parte selvaggio, con l'eccezione della sua cosa orientale, dove i primi insediamenti come Sydney sono stati inizialmente adibiti a colonie penali. Solo nel 1901 viene creata la federazione australiana che riunisce vari Stati e, pur divenendo pressoché indipendente, conserva lo status di dominion dell'Impero britannico.

I primi ebrei arrivano in Australia nel 1788 come detenuti trasportati a Botany Bay, Sydney. Molti altri arrivano poi nell'Ottocento - come Israel e Lazarus Levi con la barca Isabella nel 1818 - e alcuni di loro faranno fortuna: Solomon Levey, i fratelli Vaiben ed Emanuel Solomon, Israel Chapman, il primo detective australiano, o Isaac "Ikey" Solomon che è considerato il prototipo del personaggio Fagin descritto da Charles Dickens nel suo Oliver Twist ${ }^{9}$.

Il primo vero riconoscimento di una presenza ebraica, tuttavia, arriva nel 1817 con la nascita di una società che si occupa di sepolture ebraiche, Chera Kadisha, e la predisposizione di un apposito cimitero nel 1820. Negli anni seguenti, vengono poi fondate le prime comunità organizzate, kehilla, a Sydney (1831) e Melbourne (1841) e le prime sinagoghe, a Sydney (1844), Hobart (1845) e Melbourne (1847). Si tratta comunque di comunità molto ristrette, la cui organizzazione richiede alcuni anni di formazione: gli ebrei di Melbourne, per esempio, iniziano una propria vita associativa nel 1839 per celebrare il nuovo anno in un negozio di proprietà di Benjamin Lazarus, si organizzano ufficialmente nel 1841 con il nome di Jewish Congregational Society, associazione composta da 57 membri, e solo nel 1844 ricevono il primo grant per la costruzione della sinagoga ${ }^{10}$.

Il nucleo della comunità ebraica di origine britannica viene affiancato nel tempo da gruppi di provenienza tedesca e solo negli ultimi due decenni dell'Ottocento dagli ebrei

\footnotetext{
9 Alcune di queste vicende sono raccontate da RUTLAND, Suzan, The Edge of the Diaspora. Two Centuries of Jewish Settlement in Australia, New York, Holmes and Meier, 2001; RUBINSTEIN, Hilary L., The Jews in Australia: a Thematic History, vol.1, Melbourne, W.Heinemann, 1991; LEVI, John S., BERGMAN, George F.J., Australian Genesis: Jewish Convicts and Settlers, 1788-1850, Adelaide, Rigby, 1974.

10 «Australia’s Centenary», in Jewish Telegraphic Agency, 24 dicembre 1934.
} 
degli shtetl russi e polacchi, portatori di un ebraismo più "ortodosso", sicuramente diverso da quello praticato dai primi ebrei australiani.

L'Australia attrae i migranti perché offre spazi ancora inesplorati e selvaggi ed è un paese dove si ritiene ci si possa stanziare facilmente, approfittando delle allettanti prospettive economiche, come per le corse all'oro di fine secolo (1888, 1892-94). I nuovi arrivati portano con loro tradizioni e costumi e danno presto vita alle proprie organizzazioni religiose e culturali, che sono espressione di una realtà che a molti ebrei australiani appare come "fanatica" e radicale. A causa di ciò, si creano anche delle piccole frizioni fra gli "australiani” e le nuove comunità, che con l'aiuto di società come la Baron de Hirsch Memorial Aid Society cercano di trovare un proprio spazio, sottolineando in tal modo una divisione sempre più marcata fra le tradizionali istituzioni "anglicizzate" e i nuovi centri che sono più vicini all'ortodossia e alla cultura yiddish. I nuovi arrivati promuovono istituzioni culturali come la Kadimah di Melbourne o il teatro yiddish di Carlton, che viene lanciato da Samuel Weissberg, e movimenti di carattere socialista come la Jewish Workers' Association di Brisbane. Questa distinzione, tuttavia, non impedisce a molti emigranti russo-polacchi di fare fortuna in Australia con le loro imprese (Sidney Baevski Myer, Samuel Wynn, John Sackville, Morris Symonds di Kaunas, Adolph Basser di Cracovia). Tali persone vengono poi naturalizzate senza particolari problemi, dopo aver prestato giuramento di fedeltà e aver presentato le garanzie richieste, come accade a Samuel Berliner, nativo di Varsavia, che viene naturalizzato nel 1882 dopo solo pochi anni, o un altro polacco di Varsavia, Munn Cohen, il quale invece riceverà tale riconoscimento dopo sedici anni di residenza, nel $1891^{11}$. Le pratiche per ottenere la cittadinanza, infatti possono essere anche molto lunghe, come dimostra il caso di Sarah Cohen, vedova di Riga, la cui richiesta del 1916 (dopo sedici anni di residenza) viene ripetuta nel 1918 e include l'apposito modulo (statutory declaration), le lettere di presentazione da parte di conoscenti che garantiscono per il soggetto, la ricerca riguardante lo status del defunto marito Nathan, già residente in Australia ${ }^{12}$.

In Australia non sembra esistere lo stesso pesante antisemitismo che caratterizza la società europea, all'interno della quale all'epoca prendono piede lo scandalo Dreyfus e vengono diffusi I Protocolli dei Savi di Sion. Una qualche forma di avversione, tuttavia, appare soprattutto in relazione all'arrivo degli ebrei dalla Russia, per esempio quando il

${ }^{11}$ Entrambi secondo le norme dello stato del South Australia del 1864, secondo cui per tale richiesta occorreva presentare istanza e allegare una "garanzia" da parte di un cittadino australiano. A711, 1430, 2736.

${ }^{12}$ In tale occasione, tuttavia, si può notare una certa disponibilità da parte dei funzionari che sembrano ben disposti verso la vedova e i suoi tre figli. A1. 1916/30750; 1918/5263. 
barone Maurice de Hirsch si attiva per creare un insediamento ebraico. Dalla fine del XIX secolo, tali attitudini vengono espresse da pubblicazioni come «Bulletin» e «Truth»13. Dall'altra parte, però, la stampa mostra anche interesse per la questione ebraica in Russia, come fa il «Sydney Morning Herald» il 18 aprile 1882, riprendendo un lungo e articolato report del «Times» basato sulle notizie provenienti da agenti britannici a San Pietroburgo, Varsavia, Odessa e Taganrog. L'articolo descrive la situazione degli ebrei russi sottolineando la loro collocazione sociale e le loro tendenze professionali, che sovente sono causa di risentimento da parte della popolazione contadina: vengono quindi segnalati numerosi episodi di violenza contro le comunità ebraiche e le loro proprietà (case, negozi), ricordando le disabilities a cui gli ebrei sono sottoposti in Russia. Le conclusioni di tale rapporto indicano che la principale causa di attrito fra russi ed ebrei è di carattere economico e a tale "invidia" si è aggiunta la rabbia popolare successiva all'assassinio dello Zar Alessandro II, che le autorità hanno trattato con "apatia" e "negligenza":

The Russian authorities during these outbreaks were generally most apathetic and neglectful of their duties, allowing the brutal mobs to plunder and illtreat the Jews ${ }^{14}$.

La popolazione, d'altra parte, sembra animata da un forte spirito di fanatismo religioso che la rende cieca di fronte ai crimini che vengono compiuti sotto gli occhi di tutti, al punto che sono in tanti a negare che le violenze abbiano realmente causato delle vittime. I racconti dei rifugiati e di molti testimoni oculari, invece, appaiono quasi sempre circostanziati e sono confermati dalla documentazione presentata da consoli e diplomatici in numerose località russe.

Nel 1901, alla fondazione del Commonwealth, in Australia vivono circa 16.00o ebrei che nel 1911 diventano 17.287 e sono stanziati tanto nei grandi centri orientali come Sydney o Brisbane, dove i russi arrivano passando dalla Siberia e dalla Cina, quanto a ovest, per esempio in Western Australia, dove la comunità ebraica passa dagli 81 membri del 1891 ai 1790 del $1911^{15}$.

\footnotetext{
13 Vedi per esempio nel numero del 12 novembre 1905 del Truth, l'editoriale di John NORTON, all'interno del quale $\mathrm{i}$ pogrom russi vengono sostanzialmente giustificati in quanto: «Theologetically, ethnogenetically, ethno-psychologically, ethno-technically, and it might almost be said anthropologically, the Jews are anti-pathetic if not absolutely antagonist to the great civilised community of Christian nation of the West».

14 «The treatment of the Jews in Russia», in Sydney Morning Herald, 18 aprile 1882.

15 American Jewish Year Book Vol. 20 (1918-1919), New York, American Jewish Congress, 1920, p.341, American Jewish Year Book Vol. 3 (1901-1902), New York, American Jewish Congress,
} 
L'immigrazione in Australia viene regolamentata molto rigidamente con l'Immigration restriction act del 1901 che recepisce la politica federale della White Australia, secondo la quale si intende privilegiare l'elemento bianco possibilmente britannico o anglicizzato. L'accesso in Australia viene reso molto difficile e il perno attorno a cui ruota tale politica è il dictation test. La legge infatti prevede che venga proibito l'ingresso nel paese a chi non riesce a scrivere sotto dettatura un testo di cinquanta parole in una delle lingue europee scelta dalle autorità. Tale requisito, che rappresenta una potente arma in mano alla discrezionalità delle autorità, è stato giustamente definito la più ipocrita invenzione nella lunga storia dell'immigrazione australiana ${ }^{16}$.

Il dictation test viene infatti usato principalmente per negare l'accesso a migranti considerati "scomodi" o non assimilabili, naturalmente non sulla base di un attento esame, ma di generici pregiudizi relativi a religione, cultura o provenienza geografica. É chiaro infatti che esiste una chiara differenziazione fra gli ebrei di origine est-europea che riescono comunque a integrarsi e anche ad avere successo - e quelli anglofoni, i quali raggiungono posizioni molto importanti nella società australiana, divenendo membri delle camere legislative di alcuni Stati come Lionel Samson (in Western Australia nel 1849) e Saul Samuel (in New South Wales nel 1854), oppure distinguendosi per meriti culturali, come Max Hirsch o Sir Isaac Isaacs, o ancora nelle attività economiche (George Judah Cohen, John Sackville, Samuel Wynn) e in guerra. Durante la prima guerra mondiale, infatti, molti ebrei si mettono in luce nelle forze armate australiane, come il generale John Monash, che nel 1918 diviene comandante in capo dell'esercito australiano. Ma al di là di questa figura di primo piano, l'esercito australiano raccoglie numerosi ebrei, ormai nativi, figli di vecchi migranti che si sono stanziati da tempo e sono divenuti cittadini australiani, come Sydney Isaac Cohen che si arruola nel 1916 e cade in battaglia nel 1918, il suo quasi omonimo Cyril Sidney Cohen,

1903, p. 159. PRICE, Charles, «Jewish Settlers in Australia», in Australian Jewish Historical Society, AJHS Journal, 5, 8/1964, pp. 388-389.

16 JUPP, James, From White Australia to Woomera. The Story of Australian Immigration, Cambridge, Cambridge University Press, 2002, pp. 8-9. Chiaramente ci sono poi ulteriori divieti per malati, prostitute, ex detenuti... Per quanto riguarda il dictation test la norma recita: «The immigration into the Commonwealth of the persons described in any of the following paragraphs of this section (herein-after called "prohibited immigrants") is prohibited, namely:(a) Any person who when asked to do so by an officer fails to write out at dictation and sign in the presence of the officer a passage of fifty words in length in an European language directed by the officer» (art.3, c.A). Vedi anche WILSON, John, MCMAHON, Anthony (eds.), The Australian Welfare State, Key Documents and Themes, Melbourne, MacMillan, 1996. 
anch'egli morto in combattimento, o Solomon Michael Cohen, insegnante scolastico ferito nel 1917 e molti altri ancora ${ }^{17}$.

A cavallo fra XIX e XX secolo appaiono anche i primi movimenti sionisti, che però non hanno un appeal immediato in un paese che si dimostra tendenzialmente tollerante. Gli australiani, infatti, non sono inizialmente rappresentati ai congressi sionisti: dopo un primo tentativo (a Sydney nel 1895 nasce la Chovevei Zion Society), solo nel 1900 una associazione sionista viene fondata a Perth, nel 1902 e 1903 sorgono quelle di Sydney e Victoria, mentre nel 1908 se ne contano circa una ventina in tutto il paese.

Tali iniziative, tuttavia, vengono accolte da una generale apatia, come sottolineato nelle numerose fonti pubblicate da Marianne Dacy, o nell'appello pubblicato dallo stesso Theodore Herzl nel 1901 sulle pagine del giornale Hebrew Standard per sollecitare un supporto al Jewish Colonial Trust ${ }^{18}$. In seguito a tale appello, nel 1903 alcuni ebrei australiani, R.Beards e A.Vecht, partecipano per la prima volta al sesto congresso sionista a Basilea. Il supporto di questi e di altri movimenti sorti negli anni successivi arriva soprattutto dai migranti russi e polacchi, che sosterranno la creazione della associazione sionista Hatechiya nel 1913, sotto la leadership di Solomon Ashkenazy. Il sionismo in Australia rimane comunque una corrente estremamente minoritaria, sia per ragioni comuni ad altre parti del mondo dove viene rifiutata la secolarizzazione del messianismo, sia perché a molti appare come un tradimento della propria nuova patria, che può quindi portare a conseguenze spiacevoli.

Nel 1905 al sionismo viene data una risposta su base "territoriale" con la creazione del Jewish Territorialist Movement (JTO) da parte di Isreal Zangwill, "il Dickens del ghetto", e a queste suggestioni gli australiani replicano nel 1906, quando Richard A.Arthur e la Immigration League of Australia propongono di stabilire una colonia ebraica nel Queensland. Nel 1908 viene creata una sede locale del Jewish Territorialist Movement e A.Marks di Melbourne pianifica l'acquisto di una regione del Northern Territory, mentre nel 1910 lo stesso Zangwill pensa alla creazione di Itoland in Western Australia, suggerendo tale ipotesi al premier federale Alfred Deakin e a quello del Western Australia Newton Moore. La risposta dei due, tuttavia, è negativa e nel 1912 lo

\footnotetext{
${ }_{17}$ National Archives of Australia, collection B2455, item 3267203, vedi anche il caso di Cohen Louis Jacob, B2455, 3267165. Sulla vita di Monash, SERLE, G., John Monash: A Biography, Melbourne 1982.

18 «So we call upon you not to forget in prosperity your unfortunate brother, also not to forget that on account of the name Jew, which he wears with pride he suffers much». RUTLAND, Suzanne, The Edge of the Diaspora. Two Centuries of Jewish Settlement in Australia, New York, Holmes and Meier, p. 87. DACY, Marianne, Early Australian Zionism: an annotated index of records in the Central Zionist Archives, Sydney, University of Sydney 1993.
} 
stesso Zangwill stizzosamente dichiara che nemmeno l'Australia, pur avendo bisogno di incrementare la sua popolazione, mostra magnanimità o saggezza politica ${ }^{19}$.

Nonostante tale contrarietà, si registra comunque una interessante esperienza come quella di Shepparton, Victoria, dove nel 1913 sorge un insediamento ebraico grazie al sostegno di alcuni ebrei (Moshe A.Schalit, Isaac Jacobs, Barnet Altson e Abraham Kozminsky) e della sede di Victoria del JTO, ospitando nove famiglie delle quali molte provenienti dalla Russia (Bendall, Bereskinsky, Feiglin, Gorr, Moritz e Rubenstein).

Questo esperimento rimane tuttavia isolato in quanto la politica governativa rifiuta di prendere in considerazione l'immigrazione di gruppi ben identificati e, in base al Restriction Act del 1901, regolamenta l'immigrazione su base prettamente individualistica, considerando quindi le caratteristiche del singolo migrante. L'origine ebraica est-europea, al contrario, è motivo di diffidenza in quanto l'estraneità alla cultura britannica è ritenuta un grave impedimento per l'accettazione di nuovi arrivati, poco importa se in fuga da condizioni critiche o semplicemente alla ricerca di lavoro e di una nuova patria.

\section{La prima guerra mondiale e l'emigrazione ebraica in Australia}

Nonostante la continua fuoriuscita, è stato calcolato che nel 1914, allo scoppio della Grande Guerra, il numero di ebrei in Russia sia di circa 6 milioni e che il 95\% di essi risiedano nella Zona di Residenza. Le condizioni di tali comunità subiscono un ulteriore deterioramento durante il conflitto, in quanto il fronte russo-tedesco coincide in gran parte con la Zona di Residenza e gli ebrei sono spesso sospettati di complicità con il nemico germanico ${ }^{20}$. La guerra rappresenta per molti l'opportunità di porre fine alle sofferenza inflitte dal dominio zarista e non è dunque sorprendente che molti giornali ebraici nel mondo facciano riferimento alle speranze in una vittoria tedesca per modificare le difficili condizioni degli ebrei russi e di un paese definito semplicemente "medievale"21.

I tedeschi, d'altra parte, sono consapevoli di tale situazione, che cercano di volgere a proprio vantaggio attraverso la propaganda del comitato per la liberazione degli ebrei russi di Max Isidor Bodenheimer e la diffusione di manifesti e pubblicazioni. Gli ebrei russi, per voce del loro rappresentate alla Duma nell'agosto del 1914, Naphtali

19 «Jewish Colonization in Australia», in Daily Telegraph, 11 ottobre 1910.

20 «War Items of Special Jewish Interests», in The Sentinel, 14 agosto 1914.

21 «To make Russia a modern state builded upon righteousness and justice, may at least have a voice in the framing of the laws of that medieval country» ", in The Sentinel, 14 agosto 1914, p. 2; «Russia and the Jews», in The Sentinel, 14 agosto 1914, p. 2. 
Friedman, dichiarano però di voler rimanere fedeli allo zar e di voler prendere parte alla guerra a fianco delle altre nazionalità dell'Impero ${ }^{22}$.

Ma tali rassicurazioni non sembrano convincere appieno le alte gerarchie dell'esercito russo che emanano una serie di dure misure militari contro gli ebrei della Zona di Residenza. Tali disposizioni (deportazioni ed espulsioni forzate, cattura di ostaggi) si basano chiaramente sulla convinzione che gli ebrei siano per lo più spie o disertori pronti ad accogliere il nemico a braccia aperte e quindi inclini a collaborare con gli invasori tedeschi ${ }^{23}$.

Altre misure punitive come il divieto di usare la lingua yiddish sono ben presto accompagnate dalla violenza dei pogrom e dalla miseria crescente dei rifugiati che sono costretti ad abbandonare le proprie case e i propri averi. Le notizie riguardanti la povertà dei rifugiati e la tragicità delle condizioni della popolazione ebraica fanno il giro del mondo e suscitano la generosa reazione di numerose organizzazioni, come l'American Jewish Committee o il Joint Distribution Committee. Numerosi appelli alla solidarietà vengono pubblicati anche in Australia dove la comunità ebraica è sicuramente molto ridotta e soprattutto molto lontana dagli orrori che gli ebrei russi e polacchi vivono in prima persona.

La tragicità di tale realtà, che perdura anche negli anni successivi, genera un esodo di consistenti proporzioni, suscitando preoccupazione in tutto il mondo, in particolare nelle diverse comunità ebraiche. L'allarme è alto, tanto da generare «A cry of panic and distress», come denunciato in un appello del Joint Foreign Committee of British Deputies alla Società delle Nazioni, in cui si parla di massacri, ecatombi, devastazione di interi villaggi, crudeltà, disastri e agonie che non hanno precedenti nella difficile storia degli ebrei dell'Europa orientale. ${ }^{24}$

22 "Although we Jews have long suffered, and are still suffering, from grievous civil disabilities, we feel, nevertheless, that we are Russian citizens and faithful sons of our Fatherland». KENNAN, George, «The War and the Russian Jews», in The Outlook, 20 gennaio 1915, p. 132. LAQUEUR, Walter, A History of Zionism, New York, Tauris Parke, 2003, pp. 173-174. SZAJKOWSKI, Zosa, «The German appeal to the Jews of Poland, August 1914", in The Jewish Quarterly Review, 59, 4/1969, pp. 311-320.

${ }_{23}$ The Jews in the Eastern War Zone, New York, The American Jewish Committee, 1916, p. 65. DUBNOW, Simon, History of the Jews in Russia and Poland. From the Earliest Times until the Present Day, vol.3, Philadelphia, Jewish Publication Society, 1920; LOHR, Eric, «The Russian army and Jews: Mass Deportation, Hostages, and Violence during World War 1», in Russian Review, 6o, 3/2001, pp.404-419.

24 «The hecatombs of Proskurov, the massacres of Uman, the carnage of Fastov, the funeral pyres and devastation in hundreds of towns, the seats of ancient Jewish communities, the atrocities and cruelties inflicted, the disasters and agonies suffered, constitute a catastrophe which has no parallel in the troubled history of the Eastern Jews during recent centuries». Letter of the Joint Committee to the President of the Assembly (Geneva, December 8, 1920). 
Gli anni di guerra producono quindi importanti conseguenze, in quanto si crea il problema dei rifugiati e della sistemazione di quanti sono stati "allontanati" dalla guerra e dalla rivoluzione del 1917. Il conflitto registra inoltre la formale soppressione della Zona di Residenza e porta a un notevole incremento dei flussi migratori, che sono in gran parte costituiti dai rifugiati creati dalla guerra, dai pogrom, dalle politiche di espulsione forzata e dal caos causato dall'arrivo al potere dei bolscevichi. ${ }^{25}$ Questi gruppi di persone iniziano dei lunghi "pellegrinaggi" che cominciano nei paesi limitrofi e che spesso proseguono verso altre località considerate più "allettanti".

Tale situazione si ripercuote naturalmente anche sui paesi più lontani come l'Australia, dove la politica di accoglienza rimane molto rigida. Durante il conflitto mondiale, l'immigrazione viene infatti sottoposta a nuovi vincoli derivanti dallo stato di guerra in cui si trova il paese. La provenienza da uno stato nemico come la Germania o l'Austria-Ungheria, per esempio, comporta dei pericoli che devono essere presi in considerazione, non solo nei confronti dei pochi nuovi arrivi, ma soprattutto riguardo a chi già risiede in Australia. I documenti dell'Aliens Registration Department testimoniano i molti controlli fatti sugli stranieri provenienti da paesi nemici, come Morris Epstein, di Hobart, il quale viene controllato perché è solito organizzare riunioni "sospette", che in realtà sono solo delle semplici partite di carte. ${ }^{26}$ Di grande interesse è poi l'attività delle associazioni europee che possono risultare sospette in quanto collegate ai territori di Stati nemici, per esempio il "Jewish National Fund", con sede all'Aja, che viene monitorato in quanto ha iniziato una campagna di raccolta fondi anche in Australia (fra i suoi dirigenti ci sono tedeschi ma anche russi e americani) ${ }^{27}$.

Per quanto riguarda l'immigrazione di singoli individui, invece, la guerra non comporta alcun cambiamento nella pratica che si è consolidata dopo il 1901 e che non

25 Molte testimonianze, fra cui un documento di un ufficiale italiano che nel 1919-20 visita i territori polacchi e ucraini, sono concordi nel ritenere più umano il comportamento delle truppe bolsceviche rispetto alle bande di Petljura e ai polacchi: "queste truppe hanno lasciato nelle città e nei villaggi un ricordo peggiore di quelle bolsceviche". Relazione informativa alla data del 1 novembre 1920. Crimea e Caucaso (29 ottobre, 1920). Archivio dell'Ufficio Storico dello Stato Maggiore dell'Esercito, AUSSME, E11, 57, 18. Per quanto riguarda la situazione degli ebrei fra Polonia a Russia durante e dopo la prima guerra mondiale, cfr. BLACK, Eugene C., Squaring a Minority Triangle: Lucien Wolf, Jewish Nationalists and Polish Nationalists, in LATAWSKI, Paul, The Reconstruction of Poland, London, MacMillan, 1992; GOODHART, Arthur L., Poland and the Minority Races, London, George Allen \& Unwin Ltd, 1920; HAGEN, William H., The Moral Economy of Popular Violence. The Pogrom in Lwow, November 1918, in BLOBAUM, Robert (ed. by), Antisemitism and its Opponents in Modern Poland, Ithaca and London, Cornell UP, 2005.

${ }^{26}$ Si dichiara su mr.Epstein e i suoi ospiti: «It was ascertained that they were mostly Jews who met for the purposes of playing solo whist» (13 ottobre 1914). NAA, A3934, SC17/4, 237337.

27 «No definite grounds here for suspecting Jewish National Fund». Nota del segretario del primo ministro al ministero della difesa (27 novembre 1917). Più nello specifico viene redatto dall'Alto Commissario di Londra un report che viene allegato a un memorandum al segretario del primo ministro il 16 settembre 1918. NAA, A3934, SC17/4, 237337. 
prevede alcuna possibilità di procedere ad accettazioni "collettive" di particolari categorie, tranne nei casi gestiti attraverso la assisted migrations, quindi supportando solo l'immigrazione di cittadini britannici. Le notizie sui profughi russi e polacchi arrivano anche in Australia e il 24 giugno 1916 il «The Sydney Morning Herald» parla del grande esodo di rifugiati ebraici nell'autunno del 1915, ma anche del fatalismo di tali comunità, che accettano il loro destino con eccessiva rassegnazione ${ }^{28}$.

Ancora maggiore è l'attenzione dedicata dal «The Hebrew Standard of Australasia», che nel numero del 19 febbraio 1915 si sofferma a lungo sulla realtà del conflitto. L'editoriale «The Jews of Poland» ricorda che una buona parte dell'intera comunità ebraica mondiale è coinvolta nella tragica realtà russa e nello specifico si fa cenno all'Australia, che al pari di altri paesi, non può esentarsi dal prestare il proprio aiuto ai bisognosi:

Here in Australia we cannot well do less; for it cannot be denied that despite the smallness of our numbers we are positively the least among all the Jews of the world in feeling the adverse effects of the war. The stupendous amount of relief required by our Polish brethren makes it incumbent on every Jew in Sydney to take up the burden according to his means ${ }^{29}$.

La prima necessità è quella di informare gli ebrei australiani, perché si ritiene che una corretta e piena conoscenza di quanto accade agli ebrei in Europa orientale sia necessaria per lanciare una efficace raccolta di fondi. All'interno dello stesso numero, inoltre, è presente un altro più completo e dettagliato articolo, in cui si menzionano le iniziative benefiche dei Rothschild e si fa una stima del numero di rifugiati provenienti dall'Est Europa. A Londra se ne trovano 6-7.000, 170.00o galiziani sono a Vienna, 25.000 in Moravia e 75.00o in Boemia:

the number of refugees and destitute approximated two million - that was to say there were one-third of the Jews of Russia, absolutely destitute and starving, and this not include the extreme distress of the Jews in the Galician provinces occupied by the Russia army30.

28 "At the best of times these folk are lacking in initiative, and inclined to fatalism», in The Sydney Morning Herald, 24 giugno 1916; «The Polish refugees», in The Sydney Morning Herald, 24 giugno 1916.

29 «The Jews of Poland», in The Hebrew Standard of Australasia, 19 febbraio 1915.

3о «Two million Jews destitute. Terrible distress in Poland», in The Hebrew Standard of Australasia, 19 febbraio 1915 . 
Anche in questo caso, lo scritto si chiude con un invito a donare, a partecipare agli sforzi che tutti, a cominciare dall'Inghilterra, stanno sostenendo per aiutare la popolazione ebraica. Da parte del governo federale del Commonwealth, però, non vengono allestiti fondi speciali per l'assistenza e il soccorso delle vittime di paesi europei. Diversi governi australiani, invece, si interessano alla situazione dei profughi russo-polacchi e stanziano delle somme in loro aiuto: nell'ottobre del 1917, una inchiesta del primo ministro verifica che per il soccorso dei polacchi (fra cui rientrano chiaramente i numerosi rifugiati ebraici) il New South Wales ha stanziato 98.709 sterline, lo Stato di Victoria 7.350 e quello di Western Australia 174.16131.

I fondi suscitano comunque notevoli polemiche sulla stampa, che critica aspramente la spesa di denaro pubblico per tali finalità. Il «Bulletin» del 13 settembre 1917, per esempio, sostiene che una della cose più sbagliate che l'Australia abbia mai fatto con il denaro pubblico sia stata quella di devolverne parte a certi "rifugiati russi". Tali individui, secondo l'ottica del «Bulletin», non sono degni di tali attenzioni prima di tutto a causa del loro odio nei confronti dell'Inghilterra e della loro simpatia per il bolscevismo (descritta come odio per gli aristocratici). Con i fondi messi a disposizione - si denuncia - tali soggetti di “mentalità moscovita” («Muscovite brain») vengono messi in grado di far perdere la guerra all'Intesa ${ }^{32}$.

Un ulteriore articolo viene pubblicato sul «The Register» di Adelaide, il 20 settembre 1915, e si sofferma sulla situazione russa e sui dibattiti sull'emancipazione e la fine della Zona di Residenza. Assistere a tali dibattiti, dice, è come assistere alla battaglia delle Fiandre: da una parte la fedeltà appassionata degli ebrei tocca il cuore russo; dall'altra le forze della reazione sono ancora potenti e la guerra ha portato un'intensa ondata di sciovinismo. Un giorno si parla di riforme, quello dopo le centurie nere chiedono di estendere le restrizioni nella Galizia occupata e di confiscare tutte le proprietà ebraiche. Un giorno l'ebreo Katz è celebrato come un eroe per aver difeso la patria contro i tedeschi, il giorno dopo è espulso dall'ospedale a Pietrogrado e i giornali ne chiedono l'allontanamento dall'esercito33.

L'opinione pubblica australiana, in sostanza, viene pienamente sensibilizzata di fronte alla devastazione in Polonia e Russia che viene paragonata dal sionista Nahum Sokoloff a una seconda distruzione di Gerusalemme ${ }^{34}$. Metà della popolazione ebraica

${ }^{31}$ Comunicazione del segretariato del primo ministro al console generale in Russia (23 ottobre 1917). National Archives of Australia, A2, 1917/3354, item 47629.

$3^{2}$ Lettera del console generale in Russia del 17 settembre 1917. NAA, A2, 1917/3354, 47629.

33 ZANGWILL, Israel, "War and Jews. The Prospect of Emancipation in Russia», in The Register, 20 settembre 1915.

34 «The victims of the god of war cannot even find other houses in which to take shelter. Except at the end of an almost interminable flight. In this flight man, woman and child are taking part; 
mondiale vive in una zona di guerra in cui si confrontano i più grandi e potenti eserciti del mondo. Persone di ogni origine e rango, filantropi che sono diventati poveri dalla notte al giorno, mercanti, negozianti, lavoratori, rabbini e studenti, professionisti ed artisti sono stati cacciati dalle loro case con mogli e figli e muoiono di fame e freddo, si spostano di città in città, da villaggio a villaggio, come bestiame, senza neppure le cure che vengono date al bestiame:

In a word, a whole people are adrift from the Vistula and the Niemen to the Baltic, from the Baltic to the borders of the Volga, from the Volga to the Ural and Siberia, wandering through the ice and blizzards of the Russia winter35.

È dunque naturale che tale "calamità" produca un'enorme massa di rifugiati che trova rifugio non solo nei paesi confinanti: accade infatti che alcuni gruppi riescano a raggiungere Londra o altre capitali europee, altri compiano percorsi più complessi attraverso la Siberia e l'Asia. In tale contesto si cerca di far convergere alcuni piccoli gruppi verso l'Australia. Nella primavera del 1916, la congregazione di Brisbane intrattiene una fitta corrispondenza con il Dipartimento di affari esteri, nella quale si spiega che è consuetudine che gli europei in buona salute e in grado di guadagnarsi da vivere non siano sottoposti al dictation test, chiedendo pertanto che gli ebrei russi e polacchi possano usare il Medical Bureau e l'alto commissario di Londra in modo da ottemperare più facilmente alle richieste della legge. Il 13 aprile il presidente della Brisbane Hebrew Congregation, Philip Banner, scrive al segretario agli affari esteri, e spiega il suo progetto auspicando che le autorità acconsentano, o quantomeno non pongano eccessivi ostacoli, all'immigrazione di gruppi di ebrei in Australia. Analoghi flussi migratori stanno interessando America, Canada, Sud Africa e pertanto anche l'Australia, che dispone di spazi sconfinati, potrebbe favorire l'arrivo di coloni russi e polacchi, per esempio nel Queensland dove il governo locale sembra ben disposto:

There are hundreds of thousands of acres of waste land in Australia that are suitable for all classes of agricultural pursuits, and which might well be filled with people of the agricultural class, such as are suffering owing to the present

old and young, mother and maid, sick and well, wounded and sound-all are fugitives». «The Jewish refugees. Terrible Plight of Millions» in The Hebrew Standard of Australasia, 22 ottobre 1915 .

35 «The Russian Tragedy. Help needed», in The Hebrew Standard of Australasia, 24 marzo 1916. Altri articoli vengono poi pubblicati dal The Hebrew Standard of Australasia («Thousands of Jews expelled by Poland», 20 settembre 1918; «Persecution of Galician Jews», 24 gennaio 1919); dal Goulburn Evening Penny Post («Massacre of Jews», 3 dicembre 1918 ), o dal Northern Miner («The Bolshevik Menace», 14 aprile 1919 ). 
unfortunate European war. I believe that if assistance is given to those who are interesting themselves in England, and America that very probably they will appoint a commission to visit the various parts of the world, with a view of settling these people on the land, and I think they would be a valuable asset to our country, not alone from the productive point of view, but also from a defence point of view 36 !

Un memorandum del Dipartimento di affari esteri per il primo ministro, tuttavia, "sconsiglia" tali progetti e, considerando che sulla base dei requisiti medico-sanitari molte richieste potrebbero essere effettivamente accettate, si interroga sulla possibilità di impedire, in un modo o nell'altro, tale eventualità ${ }^{7}$. A tal fine, le autorità fanno ampio ricorso al dictation test come dimostrano alcune pressioni fatte da numerosi ebrei britannici nel 1917, i quali ritengono che tale norma venga sovente applicata in maniera inadeguata e discriminatoria ${ }^{38}$. Le basi della politica migratoria britannica e australiana, tuttavia, rimangono inalterate e così gli anni di guerra continuano a registrare cifre piuttosto limitate, anche a causa delle difficoltà causate dalle operazioni belliche su scala mondiale. Soltanto negli anni successivi arriveranno nuovi migranti dall'Europa orientale, il cui contributo apporterà significativi cambiamenti alla comunità ebraica australiana, sicuramente in modo più evidente rispetto a quanto accaduto fino alla Grande Guerra.

\section{Conclusioni}

A cavallo fra XIX e XX secolo i problemi delle comunità ebraiche in Europa orientale creano i presupposti per una grande ondata migratoria che tocca solo in minima parte l'Australia, ma che vi introduce degli elementi di novità piuttosto importanti. Fino al XX secolo, almeno secondo l'analisi fatta dalla più grande esperta del mondo ebraico australiano, Suzanne Rutland, la comunità proveniente dall'Europa orientale ha un impatto minimo sulla Australian Jewry, dominata da una leadership

${ }^{36}$ Documento del 16 maggio 1916 dell'Official Secretary del Commonwealth of Australia in Gran Bretagna, Attle Hunt, riguardante la corrispondenza con la Brisbane Hebrew Congregation inviato al dipartimento di affari esteri. NAA, A1, 1916/10708, 34455.

37 «Can we give an assurance that no departure will be made in the case of the immigrants mentioned?». Memorandum del department of external affairs del 28 aprile 1916. NAA, A1, 1916/10708, 34455 .

${ }^{8}$ Documento del 25 maggio 1917 del London committee of Deputies of the British Jews. NAA, A1, 1917, 10719, 35925. Fra la fine del 1918 e l'inizio del 1919 il fondo sionista chiede il permesso di fare partire una iniziativa per la raccolta di fondi con pubblicità anche sul «Jewish Herald». NAA, A2483, B18/7676, 151057. 
anglo-giudaica molto incline a integrarsi con la maggioranza, mentre i gruppi russopolacchi appaiono invece estremamente gelosi delle proprie tradizioni e dello loro cultura:

The Eastern European Jews who arrived before 1914 were too small in number to significantly affect the basic attitudes of Australian Jewry and were themselves often influenced by pressures to conform to the ways of their new country. This weakened group cohesion threatened the continued existence of the community as a distinctive entity39.

La crescita di questa nuova comunità viene incrementata proprio dagli eventi della prima guerra mondiale e dal caos che regna in Russia dopo le rivoluzioni del 1917. Tale flusso verrà però visto negativamente da quanti esprimono il timore di vedere la propria posizione danneggiata dalla presenza di nuovi arrivati poco "civilizzati" che rischiano di arrecare un danno alla floridezza della comunità ebraica australiana ${ }^{40}$.

Gli ebrei est-europei apportano notevoli cambiamenti alla realtà ebraica australiana di origine anglofona, che si era andata progressivamente integrando, rinunciando ai caratteri più tradizionali della propria identità. I migranti che arrivano dall'Est Europa, al contrario, mantengono una forte radice yiddish e una religiosità più ortodossa, distinguendosi così dalla maggioranza. Come ha sottolineato Oscar Handlin, l'attaccamento alle proprie tradizioni "ancestrali" è un fenomeno piuttosto comune per un emigrante, il quale percepisce una nuova realtà per i suoi "pericoli”, consolidando negli "sradicati" il bisogno di stringersi attorno alla propria fede e coltivare la propria cultura $^{41}$. Anche a causa di questa naturale "chiusura", l’immigrazione di ulteriori ebrei viene solitamente supportata dai gruppi più "giovani", mentre l'establishment anglofono tende a far propria la politica ufficiale della White Australia, prendendo così le distanze dai nuovi arrivati e dal loro forte attaccamento a tradizioni religiose radicate soprattutto in campo educativo e culturale ${ }^{42}$.

39 RUTLAND, Suzanne, The Edge of the Diaspora, cit., p. 106.

40 "We Australian Jews in this remote outpost of the British Empire are Britishers to the backbone and spinal marrow». Dichiarazione pubblicata sul «Jewish Herald» (21 marzo 1919). Sul ruolo degli ebrei nel mondo politico australiano, cfr. BRAHM LEVEY, Geoffrey, MENDES, Philip (ed.by), Jews and Australian Politics, Brighton, Sussex Academic Press, 2004.

${ }^{41}$ «In the American environment, so new and so dangerous, these people felt more need than ever for the support of their faith». HANDLIN, Oscar, The Uprooted. The Epic Story of the Great Migrations that made the American People, Boston-Toronto, Little, Brown \& co., 1973, p. 127.

42 In uno studio dedicato all'analisi comparata dei flussi ebraici in Argentina, Sud-Africa e Australia, si sottolinea l'impatto delle comunità arrivate dall'Est Europa nel dopoguerra: «Thus, Australia came to be the only one of the new societies in which a majority of the migrants came 
Il caso australiano mostra come, nonostante l'esistenza di presupposti favorevoli la scarsità della popolazione australiana, gli enormi spazi a disposizione, l'assenza di un diffuso antisemitismo - l'arrivo di nuovi migranti sia accompagnato dalla generale opposizione di governo e opinione pubblica, che non rispecchia i canoni dei tradizionali pregiudizi antisemiti e viene invece alimentata da un forte sentimento di ostilità verso tutto ciò che appare estraneo all'elemento anglofono che ha forgiato la società australiana fino al XX secolo. Sembra paradossale, comunque, constatare come le comunità ebraiche che in Europa orientale vengono percepite attraverso immagini stereotipate di speculatori, sfruttatori, elementi "cosmopoliti" estranei alla società russa, in Australia siano invece stigmatizzate per la loro "inferiorità" e la provenienza da un mondo "barbaro" e sottosviluppato. Non hanno nessuna influenza ragionamenti di tipo umanitario o discorsi di natura politica attinenti alla particolare situazione dell'Europa orientale e piuttosto ridotta appare anche la disponibilità delle comunità ebraiche australiane, che non sembrano volersi impegnare se non attraverso raccolte di fondi o iniziative di carità.

Il rifiuto delle autorità di collaborare a qualsiasi progetto di colonizzazione, la "fermezza" con cui si applica il dictation test a determinate categorie di migranti - fra cui rientrano per l'appunto gli ebrei est-europei - e la refrattarietà con cui il governo federale accoglie le iniziative di solidarietà dei singoli Stati federati mostrano come l'immigrazione in Australia segua un percorso specifico, finalizzato a mantenere l'originale carattere britannico della società australiana. Le vicende dei migranti ebraici dopo la fine del conflitto e, in particolare, in seguito all'avvento del Nazismo in Germania, renderanno ancora più evidente la consistenza di un particolare etnocentrismo australiano. I pregiudizi nei confronti della diversità hanno dimostrato di poter essere ben dissimulati attraverso strumenti come il famigerato dictation test e di potersi confondere con questioni di ordine sociale ed economico, creando profonde divisioni anche fra le comunità ebraiche australiane, le quali sono sì unite dalla comune religione, ma nel XX secolo cominciano ad apparire profondamente divise in base a estrazione culturale, orientamenti politici o provenienza geografica.

with strong prior commitments to creating a traditionally oriented Jewish community. They immediately established educational and religious institutions for this purpose, and directed all their communal efforts to that end, turning to the other previously established communal institutions only when it became apparent that influence within them would be useful for advancing their basic goal». ELAZAR, Daniel J., «Jewish Frontier Experiences in the Southern Hemisphere: The Cases of Argentina, Australia, and South Africa», in Modern Judaism, 3, 2/1983, pp. 129-146, p. 136. 


\section{* L'autore}

Giuseppe Motta è attualmente ricercatore in Storia dell'Europa orientale presso Sapienza Università di Roma ed è membro del comitato esecutivo dell' "Istituto italo-romeno di studi storici” (Cluj Napoca, Romania) e del collegio docenti del dottorato in Storia d'Europa dell'università Sapienza. Ha lavorato inoltre presso le università Babes-Bolyai di Cluj (Romania), di Mostar (Bosnia-Erzegovina), Viterbo e Bergamo, svolgendo un periodo di ricerca grazie al programma europeo Basileus presso l'università di Sarajevo (Bosnia-Erzegovina). La ricerca nel corso degli ultimi anni si è concentrata sullo studio delle minoranze nazionali in Europa centro-orientale, tema a cui sono stati dedicati numerosi articoli e le monografie: Le minoranze nel XX secolo. Dallo Stato nazionale all'integrazione europea, Milano, FrancoAngeli, 2006; The Italian Military Governorship in South-Tyrol and the Rise of Fascim, Roma, NuovaCultura, 2012; Less than Nations. Central-Eastern European Minorities after WW1, 2 voll., Newcastle, Cambridge Scholars Publishing, 2013; Robie. La schiavitù dei rom in Valacchia e Moldavia, Roma, Aracne, 2013; The Legacy of the First World War. The Minority Question in Transylvania, Targu Mures, Petru Maior University Press, 2014.

URL: < http://www.studistorici.com/progett/autori/\#Motta >

\section{Per citare questo articolo:}

MOTTA, Giuseppe, «Le comunità ebraiche in Australia e gli arrivi dall'Europa orientale fra XIX e XX secolo», Diacronie. Studi di Storia Contemporanea : Costruire. Rappresentazioni, relazioni, comunità, 29/06/2015, URL:<http://www.studistorici.com/2015/06/29/motta_numero_22/ >

\section{Diacronie Studi di Storia Contemporanea $\widehat{\beta}$ www.diacronie.it}

Risorsa digitale indipendente a carattere storiografico. Uscita trimestrale. redazione.diacronie@hotmail.it

Comitato di redazione: Jacopo Bassi - Luca Bufarale - Elisa Grandi - Deborah Paci - Fausto Pietrancosta - Matteo Tomasoni - Luca Zuccolo 